\title{
Scalable modulation for scalable wireless videocast
}

\author{
Lin Cai, Yuanqian Luo, Siyuan Xiang, and Jianping Pan \\ University of Victoria, Victoria, BC, Canada
}

\begin{abstract}
In conventional wireless systems with layered architectures, the physical layer treats all data streams from upper layers equally and apply the same modulation and coding schemes. Newer systems such as Digital Video Broadcast start to introduce hierarchical modulation schemes with SuperPosition Coding (SPC) and support data streams of different priorities. However, SPC requires specialized hardware and has high complexity beyond most existing handheld devices. We thus propose scalable modulation (s-mod) by reusing the current mainstream modulation schemes with software-based bit-remapping. In this paper, we study how to optimize the configuration of the physical layer s-mod and coding schemes to maximize the utility of videocast with Scalable Video Coding (SVC). Simulation results demonstrate significant performance gains using s-mod and the cross-layer optimization, indicating s-mod and SVC is a good combination for wireless video multicast and unicast.
\end{abstract}

\section{INTRODUCTION}

In current wireless systems, the physical (PHY) layer treats every bit from the upper layer with equal priority. However, for some emerging applications, bits from the same application may have different importance and impact on the user-perceived Quality-of-Services (QoS). For instance, the Scalable Video Coding (SVC) schemes may encode the video streams into a base layer and an enhancement layer, where packet losses in the base layer lead to much severer QoS degradation than the losses in the enhancement layer. How to fine-tune the PHY layer, especially the modulation scheme, to more efficiently support layered, scalable video is an important and challenging issue. SuperPosition Coding (SPC) and its implementation (i.e., hierarchical modulation, or h-mod) has been considered as a promising candidate for video multicast. However, SPC (h-mod) requires more complex hardware, so its implementation cost in the current wireless systems such as $3 \mathrm{G}$ and WiMAX is prohibitively high. In addition, the crosslayer problem of how to optimize the configuration of the PHY layer modulation and coding schemes to maximize the utility of scalable video multicast is an open issue.

To provide differentiated services to upper-layer data streams, we propose scalable modulation (s-mod) schemes, which can be implemented using the existing Quadrature Amplitude Modulation (QAM) hardware. With the softwarebased approach, the s-mod schemes just redefine (or remap) the constellation points of the existing QAM to modulate and demodulate the layered bits with different bit error probabilities (or Bit Error Rate, BER). In this paper, we formulate a cross-layer optimization problem, aimed to maximize the profit (which equals the utility minus the cost) of scalable video multicast, by optimizing the configuration of the PHY s-mod and coding schemes. Extensive simulations with real videos and realistic wireless channel profiles are conducted to evaluate the system performance and demonstrate the advantage of the proposed cross-layer optimization framework and the s-mod schemes.

The rest of the paper is organized as follows. Sec. II introduces the related work about SPC/h-mod, scalable video coding, and cross-layer optimization for supporting scalable videos. Sec. III overviews the s-mod schemes proposed by us. The cross-layer optimization problem is formulated in Sec. IV. Performance evaluation by simulation is given in Sec. V, followed by concluding remarks in Sec. VI.

\section{PRELIMINARIES AND RELATED WORK}

\section{A. Superposition coding and hierarchical modulation}

For time-varying wireless channels, Adaptive Modulation and Coding (AMC) schemes have been proposed and widely deployed in wireless systems. However, AMC is not suitable for multicast or broadcast transmissions, as different receivers have different channel conditions and prefer different modulation and coding schemes. Consider the scenario that a Base Station (BS) multicasts to a group of receivers in the downlink. To ensure the reliable transmission of all bits to all receivers, the BS has to choose the modulation and coding schemes according to the user with the worst channel condition at any time. On the other hand, for many multicast applications, such as scalable video streams, receivers can enjoy the services even if only part of the data are received successfully. For these applications, hierarchical modulation (h-mod) schemes based on SuperPosition Coding (SPC) have been proposed [1].

The idea of h-mod (SPC) is to combine the signals for multiple layers of the data streams into one single symbol stream, where symbols for different layers are synchronously superimposed together before transmission [2]. Receivers with good channel quality may demodulate bits from all layers successfully; those with bad channel quality may demodulate part of the bits only. From an information-theoretical perspective, SPC can achieve higher maximum sum rate of Gaussian broadcast channel than the time-sharing schemes (i.e., modulating and transmitting bits of different layers separately). However, existing h-mod schemes require specialized hardware for interference cancellation, and they suffer from Inter-Layer Interference. Thus, although h-mod has been adopted in the Digital Video Broadcast standard, it is difficult to be used in wireless networks due to the hardware and performance limits. This motivates us to propose the low-complexity softwarebased scalable modulation schemes.

\section{B. Scalable video coding}

Scalable Video Coding (SVC) is an emerging, appealing coding technique for video streaming and IPTV applications. It has been included in the H.264 standard. SVC provides spatial, temporal, and quality scalability. Sub-bitstreams with different type of scalability can be extracted from a single SVC encoded 
bitstream. When different subsets of the bitstream are decoded, videos with different frame rate, resolution and quality can be reconstructed. This feature is naturally more efficient than simulcast or transcoding for heterogeneous network users. Layered structure is essential for SVC. For each type of scalability, there is one base layer and one or more enhancement layers. SVC exploits the correlation between the base layer and the enhancement layers (higher layers) to reduce the data rates of higher layers. Taking the two-layer coarse-grained quality scalability (CGS) as an example, encoded bitstreams can be divided into two layers. The base layer provides the minimum satisfactory video quality; with the enhancement layer, better video quality can be achieved. However, when the base layer is corrupted or lost, the enhancement layer itself cannot improve the video quality or even decode the video bitstream. When we choose modulation and coding schemes, it is desirable to provide different services for different layers. This is another motivation for our proposed s-mod schemes.

\section{Cross-layer optimization for wireless video streaming}

Resource allocation for supporting video streaming over wireless networks is challenging, due to the time-varying wireless channels and the highly bursty video traffic. Existing work [3]-[6] mainly focused on the link layer and above, and did not consider how to manage the PHY layer schemes to support layered videos. Recently, cross-layer optimization for wireless multicast considering AMC began to attract attention. In [7], [8], how to choose the right modulation and coding schemes to ensure efficient and reliable multicast was studied. How to optimizing power allocation for SPC was proposed in [9]. Different from the previous work, in this paper, we first propose s-mod schemes that can support differentiated services and layered videos without involving specialized hardware. Then, we propose a cross-layer optimization framework to configure PHY layer transmission schemes appropriately, aimed to maximize the profit of the videocast services.

\section{SCAlABLE MODUlation (S-MOD)}

The main objective for s-mod is to efficiently provide differentiated services for different classes of information bits. To achieve service differentiation, we can construct the signal constellation of the modulation scheme such that the Minimum Euclidean Distances (MEDs) of the symbols in different layers are different. Another design constraint for wireless systems (3G or WiMAX) is the relatively simple transceiver design of handheld devices. The complexity of demodulation makes the h-mod technology not readily deployable in portable devices. Thus, we aim to design scalable modulation schemes that can be used with existing modulation/demodulation hardware.

In the PHY layer of wireless systems, typically QAM schemes are supported. We can use software to map the signal constellations of the existing QAM modulation schemes to support differentiated services. Fig. 1 gives a simple example to demonstrate the advantages of the s-mod schemes.

Fig. 1(a) shows a proposed software-based s-mod scheme, which supports two-layer bits with the MED of $2 d$ and $d$ for

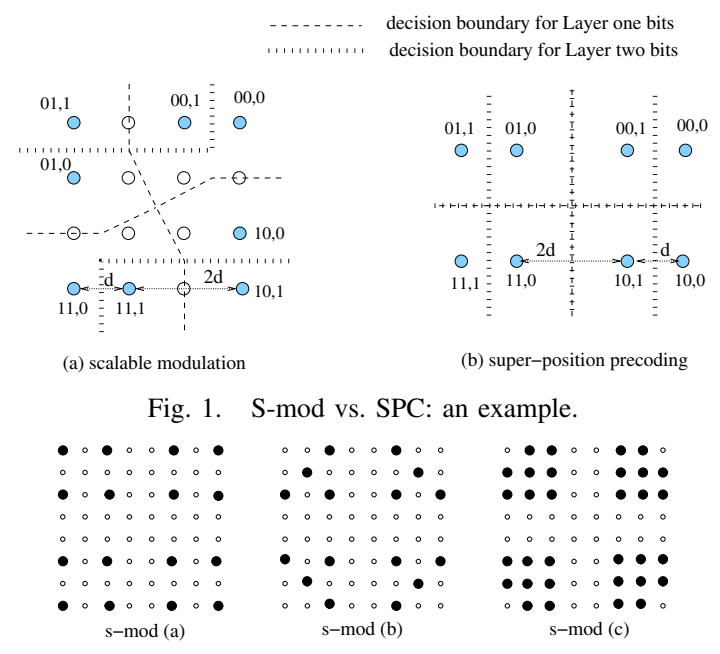

Fig. 2. S-mod schemes for two-layer bit-mapping.

layer-one (L1), the high priority layer, and layer-two (L2), the low priority layer, respectively. The s-mod scheme is compared with the traditional SPC scheme shown in Fig. 1(b). The bit-energies of both schemes are the same, equal to $3.5 d^{2}$. The demodulation decision regions can be obtained by finding the Voronoi diagram of the signal space for each layer. The probability of each layer's bits can be successfully demodulated equals the probability that the received signal is within their decision region. Given an additive white Gaussian noise (AWGN) channel, the BERs for the two layers with smod are both lower than those with SPC. For instance, with the received SNR equal to $10 \mathrm{~dB}$, error probability $e_{1}=0.010$ and $e_{2}=0.116$ for the s-mod scheme, and $e_{1}=0.013$ and $e_{2}=0.120$ for the SPC, where $e_{1}$ and $e_{2}$ are the BERs for L1 and L2 bits, respectively. The s-mod scheme can outperform the traditional SPC (h-mod) thanks to its flexibility in arranging symbol constellations, so the decision regions for both layer bits can be larger.

The procedures to construct s-mod schemes and the details on calculating the BERs for bits from different s-mod layers are referred to [10]. Fig. 2 shows three examples of the smod schemes for two-layer bit-mapping. Note that some s-mod schemes are equivalent to the SPC (h-mod) schemes, such as the one shown in Fig. 2(a). However, s-mod has more choices in the arrangement of constellation points than SPC, such as those in Figs. 1(a), 2(b) and 2(c).

\section{CROSS-LAYER OPTIMIZATION WITH S-MOD}

\section{A. System model}

We consider an infrastructure-based wireless system. A BS covers the area of a cell, and it can multicast videos to the mobile stations (MSs) within the cell in the downlink. The video streams are coded with a layered SVC codec. Concatenated Reed-Solomon (RS) and convolutional coding with interleaving have been used to enhance the BER performance in existing wireless systems, and they are adopted in our system model. The layered video streams are encoded by an RS encoder and interleaved before being fed to a convolutional encoder. At the receiver side, the procedures 
are simply reversed. The concatenated RS and convolutional code can effectively reduce the BER, which is essential for the QoS guarantee of video applications. Given the two-layer s-mod scheme, the ratio of link-layer data rates of the two bit-layers is given, which might be different from the ratio of the data rates of the coded layered video streams. The leftover bits from one of the layers will be fed to an s-mod mapping for single-layer bits [10]. This s-mod scheme is chosen such that the BER of the leftover bits is close to that of the other bits from the same layer.

In the proposed system, the s-mod and coding schemes can be changed according to user requirements and channel conditions. However, for multicast applications, it is very difficult if not impossible to track the instantaneous channel conditions for all users. Practically, multicast receivers (the mobile stations) report their channel conditions back to the sender (the base station) periodically. Thus, the sender needs to decide the best modulation and coding schemes based on the rough estimation of time-average channel conditions. Other network protocols like the medium access control, routing, transport layer ones, also affect the QoS, particularly the delay, which has been heavily investigated before and is ignored in this work. We then assume all video packets can be delivered timely to the receiver.

\section{B. Optimal configuration problem formulation}

The main objective of our work is to maximize the utility of video multicast service and minimize the cost to provide the service, i.e., to maximize the profit (utility minus cost) of such service.

Consider a multicast group with $N$ users: $\mathbf{W}=$ $\left\{w_{1}, w_{2}, \ldots, w_{N}\right\}$, and the average received SNR of user $w_{i}$ is $\gamma_{i} \in \boldsymbol{\Gamma}$, where $\boldsymbol{\Gamma}$ is the range of the received SNR. Consider a two-layer video stream with data rates $r_{1}$ and $r_{2}$ for L1 (the base layer) and L2 (the enhancement layer), respectively. Let $m, m^{\prime} \in \mathbf{M}$ be the s-mod schemes for the two-layer bits and the leftover single-layer bits, respectively, and $c_{1}, c_{2} \in \mathbf{C}$ be the coding schemes that are chosen for the two-layers. Given the s-mod and coding schemes and the data rate of the video stream, we can estimate the bandwidth (BW) needed to deliver the two-layer video streams: $B W=$ $f_{1}\left(m, m^{\prime}, c_{1}, c_{2}, r_{1}, r_{2}\right)$. Each s-mod $m$ symbol represents $x_{1}$ L1 bits and $x_{2}$ L2 bits and each s-mod $m^{\prime}$ symbol represents $x_{3}$ bits. $c_{1}$ and $c_{2}$ has the code rate of $y_{1}$ and $y_{2}$, respectively. The baud rate (which is proportional to $B W$ ) of the signal is $\min \left(\frac{r_{1}}{y_{1} x_{1}}, \frac{r_{2}}{y_{2} x_{2}}\right)+\left|\frac{r_{1}}{y_{1} x_{1}}-\frac{r_{2}}{y_{2} x_{2}}\right| \frac{x}{x_{3}}$, where $x=x_{1}$ if L1 bits left and $x=x_{2}$ otherwise.

For $w_{i}$, given $\gamma_{i}$ and the channel fading model, we can obtain the BERs of the two layers and those of leftover bits, $e_{1}^{i}=f_{2}\left(m, c_{1}, \gamma_{i}\right), e_{2}^{i}=f_{3}\left(m, c_{2}, \gamma_{i}\right)$, and $e_{l}^{i}=$ $f_{4}\left(m^{\prime}, c^{\prime}, \gamma_{i}\right)$, respectively, where $c^{\prime}$ equals $c_{1}$ (or $c_{2}$ ), if the leftover bits are from L1 (or L2) [10]. The utility describes the satisfaction level of the user, and it directly reflects the video QoS. We can map the PHY layer performance index (BER of each layer) to the network performance index (packet loss rate of each layer), and then map to the utility. Thus, the utility of $w_{i}$ depends on its BER performance of the two layers: $U_{i}=f_{5}\left(e_{1}^{i}, e_{2}^{i}, e_{l}^{i}\right)$. Sec. $\mathrm{V}$ will give a sample mapping of $f_{5}$.

To consider both the utility and the BW cost for profit calculation, we set a parameter $\tau$, which is the unit cost of BW over the unit price of utility, and it is determined by network operators. The objective is to find the optimal modulation and coding configuration such that the profit of the video service, $P=\left\{\sum_{i=1}^{N} U_{i}-\tau B W\right\}$, can be maximized, the total $\mathrm{BW}$ allocated is bounded, and each user obtains at least $U_{\text {min }}$ utility. This is an NP-hard combinatorial optimization problem.

\section{Algorithm for searching the optimal configuration}

There is no polynomial time algorithm to obtain the optimal solution for the combinatorial optimization problem. However, since the number of choices (combinations of modulation and coding) is very limited, we can use a simple searching algorithm to find the optimal configuration. To make it feasible for realtime optimal configuration, we divide the task into two parts. The first part is using Algorithm 1 to create lookup tables, which is relatively time-consuming (a few seconds) and can be done offline. The second part is to use Algorithm 2 to search the optimal solution using the lookup tables, which has low computational complexity and can be done in realtime.
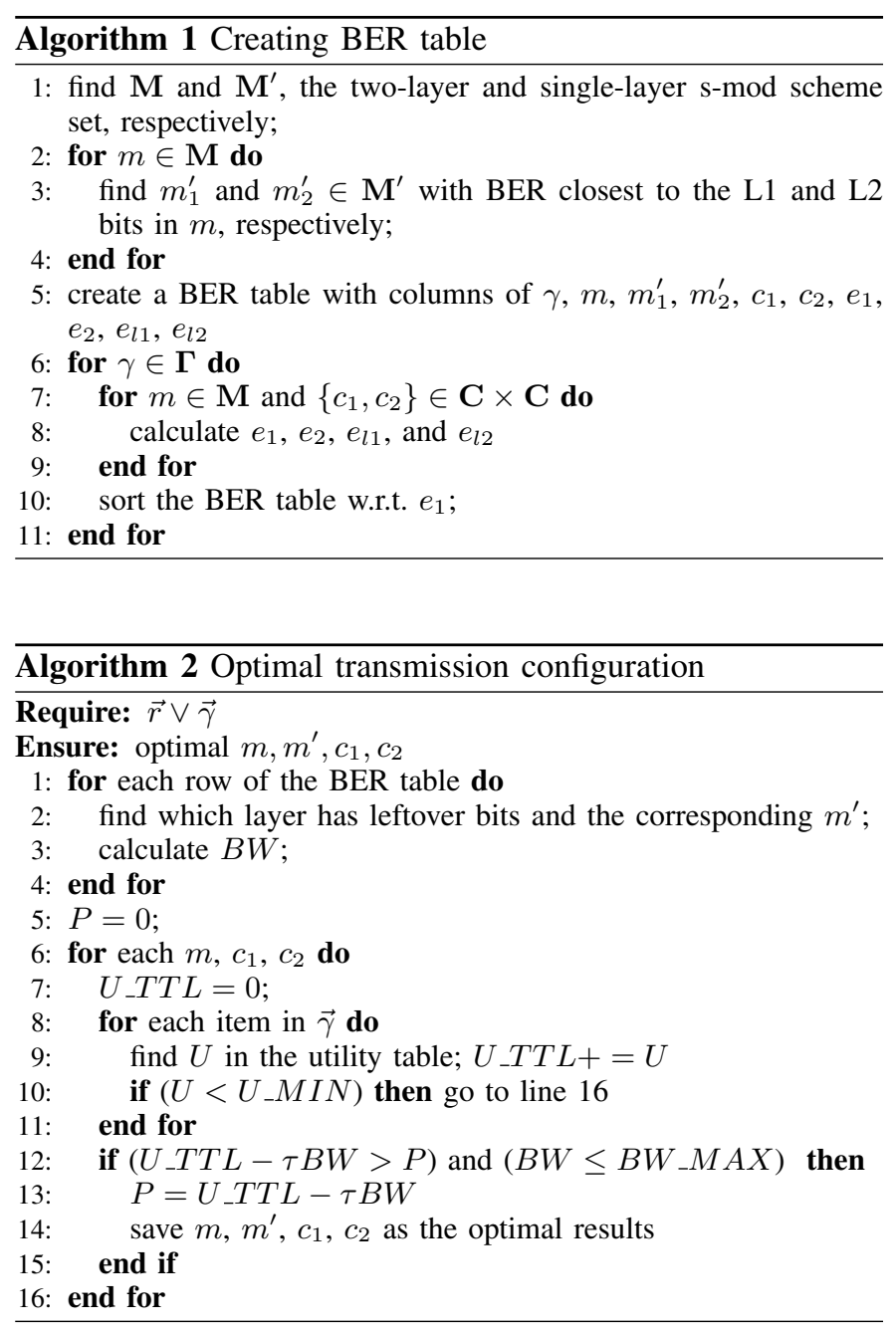


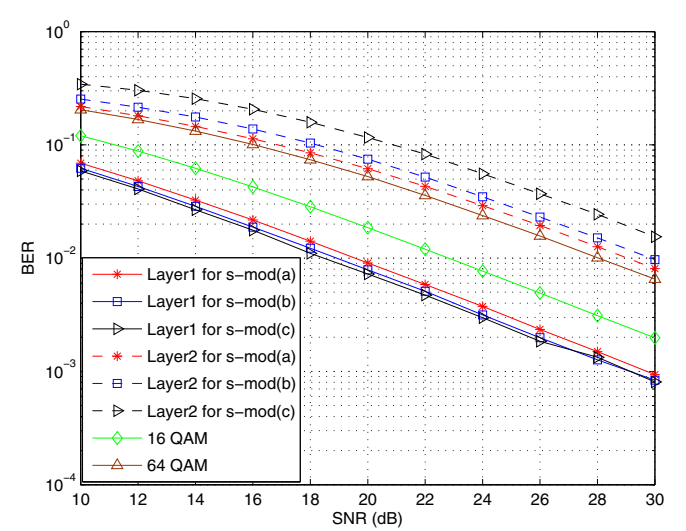

Fig. 3. BERs with different modulation schemes.

For Algorithm 2, the input variables $\vec{r}=\left[\begin{array}{ll}r_{1} & r_{2}\end{array}\right]$ are the data rate of the layered video, $\vec{\gamma}$ are the SNRs of all users. The first 4 lines of Algorithm 2 need to be executed for each video stream once it is admitted. Since the cardinalities of $\mathbf{M}$ and $\mathbf{C}$ are limited, this procedure is manageable during the admission process. The optimal configuration needed to be executed in realtime is Line 5 to 16 . Since this part involves looking up the sorted BER and utility tables once per configuration, the time needed to get the optimal solution is $O(n \log (n))$ only, where $n$ is the number of rows in the tables. As $n$ is not a large number, the optimal configuration can be done in realtime.

Claim 1: With more receivers of the multicast service, the optimal configuration tends to choose lower BER modulation and coding schemes.

Claim 2: With more low-channel-quality receivers of the multicast service, the optimal configuration tends to choose lower BER modulation and coding schemes.

The two claims above can be explained as follows. In general, a lower BER configuration results in higher utility per user and requires more BW. For multicast services, once the transmission configuration is given, the difference of $\mathrm{BW}$ costs of two different schemes is fixed no matter how many receivers there are, but the difference of the utility increases w.r.t. the number of receivers, especially when there are more low-channel-quality receivers. Thus, the number of receivers and their channel quality distribution have a great impact on the transmission configuration.

\section{PERFormance EVAluation}

\section{A. s-mod performance evaluation}

We first evaluate the PHY layer performance of the smod schemes. We simulate Rayleigh fading channels with the received SNR varying from $10 \mathrm{~dB}$ to $30 \mathrm{~dB}$, and measure the BERs of different layers after demodulation, without using error coding. Fig. 3 shows the BERs of L1 and L2 bits for the s-mod schemes, 16-QAM and 64-QAM, respectively. The s-mod schemes (a), (b) and (c) are those shown in Fig. 2. For 16- and 64-QAM, both layers have the same BERs. With the s-mod schemes, we can achieve lower BER for L1 than that of 16-QAM, while the BER for L2 is higher than that of 64-QAM. In the following subsections, we will demonstrate the advantage of such service differentiation for layered video

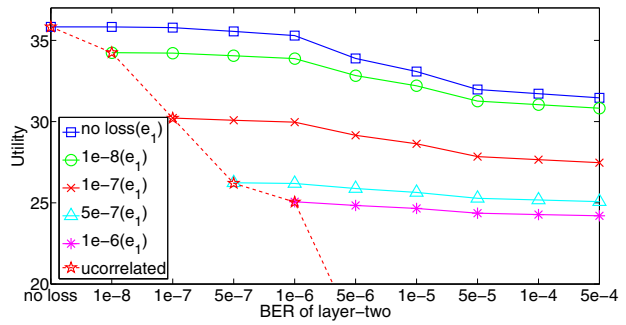

Fig. 4. Utility function of "Foreman" for single user

multicast. Among the three s-mod schemes, s-mod (c) has the lowest BER for L1 and it is most bandwidth efficient as it can transmit three L2 bits per symbol. The performance of s-mod (a) is the same as that of SPC.

\section{B. Utility of video services}

Peak signal-to-noise ratio (PSNR) reflects the distortion of the video and is frequently used as a video quality metric. Denote by $F$ the total number of frames in a video sequence, $M$ the number of pixels in a frame, $X_{i j}$ and $Y_{i j}$ the value of the $j$-th pixel in the $i$-th original and reconstructed frames, respectively. PSNR is calculated by $10 \log _{10} \frac{M \times 255^{2}}{\overline{s s d}}$, where $\overline{s s d}=\frac{1}{F} \sum_{i=1}^{F} \sum_{j=1}^{M}\left(X_{i j}-Y_{i j}\right)^{2}$.

With layered video coding, losses of L2 packets result in higher $\overline{s s d}$ and degraded PSNR, and losses of L1 packets result in not only PSNR degradation, but also sometimes undecodable frames. To measure the user's satisfaction level, the utility function is defined as the product of PSNR (of the decoded frames) and the decodable rate. The decodable rate is the ratio of the number of successfully decoded frames over the number of total frames.

We give a sample utility function (obtained by simulation) of a video "Foreman" in Fig. 4. The 20 second video has a resolution of $352 \times 288$ and a frame rate of $30 \mathrm{frame} / \mathrm{sec}$. It is coded using the Joint Scalable Video Model. The encoder is configured as two-layer CGS, and the decoder discards frames with any error bits. The base layer quality (in terms of PSNR) is around $30 \mathrm{~dB}$, and the quality gap of the base layer with or without the enhancement layer is about $4 \mathrm{~dB}$. The bit rates of the base layer and the enhancement layer are $148.16 \mathrm{Kbps}$ and $522.84 \mathrm{Kbps}$, respectively. In Fig. 4, each solid line represents the utility with the same $e_{1}$, and the horizontal axis is $e_{2}$. When $e_{2}$ is larger than $5 \times 10^{-4}$, almost all L2 frames are lost and the utility remains the same. The utility function of 16-QAM and 64-QAM is slightly different from that for the s-mod schemes, since bit-errors of L1 and L2 are equal and less correlated. Dotted line "uncorrelated" represents the utility function for 16- and 64-QAM with uncorrelated and identical bit errors for both layers. As shown in Fig. 4, the correlation of bit errors in the two layers has minor impact.

\section{Video performance of single receiver}

We investigate the utility of a single user with received SNR of $12 \mathrm{~dB}$. We fix the modulation schemes, 16-QAM, 64QAM, or s-mod scheme (b), and use the optimal configuration algorithm to find the best RS and convolutional codes for it. We use $\operatorname{RS}[255,191]$ and convolutional code with rate $1 / 2$ 


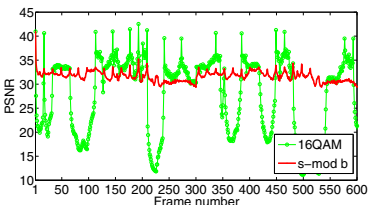

(a) PSNR

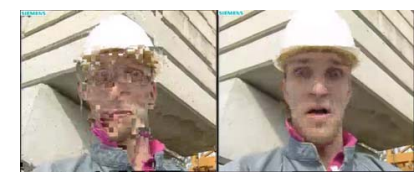

(b) 16-QAM vs. s-mod
Fig. 5. Compare 16-QAM and s-mod (b).

TABLE I

PROFIT COMPARISON

\begin{tabular}{|c||ccccc|}
\hline case & s-mod a/SPC & s-mod b & s-mod c & 16QAM & 64QAM \\
\hline \hline uni-5 & 132.5956 & $\mathbf{1 3 3 . 9 8 3}$ & 132.023 & 79.990 & 46.089 \\
\hline uni-15 & $\mathbf{4 5 8 . 1 7 6}$ & 457.257 & 450.911 & 383.931 & 185.128 \\
\hline bad-5 & 124.133 & 126.289 & $\mathbf{1 2 9 . 2 3 6}$ & 44.159 & -24.478 \\
\hline good-5 & 136.799 & 135.164 & 132.996 & $\mathbf{1 3 7 . 4 2 5}$ & 77.633 \\
\hline excel-5 & 149.048 & 148.307 & 141.758 & 142.440 & $\mathbf{1 5 3 . 5 8 1}$ \\
\hline
\end{tabular}

for 16-QAM and the L2 bits of the s-mod scheme, and use RS [255, 205] for the L1 bits of the s-mod scheme. With 16-QAM, $e_{1}$ and $e_{2}$ are in the order of $10^{-6}$. With a nonnegligible loss rate of the base layer, the decodable rate is only 0.9 , and the PSNR is very low when the base layer frames are lost. With 64-QAM, the BERs are so high that nothing can be decoded. With the s-mod scheme, the base layer frames are all received successfully, so the decodable rate is one, and the PSNR is stable although $e_{2}$ with s-mod is higher than that with 16-QAM.

Fig. 5 (a) compares the PSNR with 16-QAM and the s-mod (b) scheme. The random screen shots of the videos are given in Fig. 5 (b). With 16-QAM, the PSNR variation is significant and the video quality may degrade frequently, which results in an annoying viewing experience for the video user. The video quality is quite stable and acceptable with the proposed s-mod scheme. The simulation results demonstrate the importance of providing differentiated services for layered video streams and the advantage of the proposed s-mod scheme.

\section{Video utility of multicast receivers}

Next, we investigate the total profits for a group of multicast receivers. We consider different scenarios: groups of 15 or 5 users with received SNR uniformly distributed in $[10,22] \mathrm{dB}$ (uni-15 or uni-5), and groups of 5 users with bad, good, or excellent channels, respectively (bad-5, good-5, or excel-5). The bad-5, good-5, and excel-5 groups have SNR distributed in $[10,14] \mathrm{dB},[14,22] \mathrm{dB}$, and $[20,22] \mathrm{dB}$ ranges, respectively. For the s-mod schemes, the leftover bits of the enhancement layer are modulated using 64-QAM, so the total bandwidth required using s-mod combined with 64-QAM is smaller than that using 16-QAM only, and also smaller than that using 16QAM for L1 bits and 64-QAM for L2 bits. $\tau$ is set to 0.1 (dB·sec/kilo-symbol). For each modulation scheme, the error codes leading to the highest profit are chosen.

The profits of different schemes are compared in Table I. For different scenarios, we should choose different modulation schemes to maximize the profit. In specific, when some of the users' channel quality is bad, the s-mod schemes are preferable, as both 16- and 64-QAM require higher SNR for L1 bits. Comparing the uni-5 and uni-15 scenarios, although the channel quality distributions are the same, the optimal modulation schemes are different. This is because s-mod (a) (or SPC) needs to be coupled with stronger error coding schemes and its bandwidth requirement is higher than that of s-mod (b), and the utility of SPC or s-mod (a) can be better than that of s-mod (b) when the SNR is around $18 \mathrm{~dB}$. (Below $18 \mathrm{~dB}$, L2 frames cannot be successfully decoded with both s-mod (a) and (b); and above $20 \mathrm{~dB}, \mathrm{~L} 2$ can be decoded with both schemes.) Therefore, only when the number of users is higher, s-mod (a) (SPC) is preferable. This observation echoes our Claim 1 in the previous section. The results also verify our Claim 2: when the group of users have bad channel quality (bad-5), the optimal solution is to use s-mod (c), which has the lowest BER for L1.

The optimal configuration will choose 16-QAM if all users have good channel quality, and choose 64-QAM only if all users have received SNR above $20 \mathrm{~dB}$. Nevertheless, in these cases, the performance of the s-mod schemes is still very close to the optimal. In summary, the proposed s-mod schemes provide many more choices in the PHY layer to effectively support scalable video multicast. Combined with optimal configurations, we can achieve much higher profit for service providers and ensure better video quality.

\section{CONClusions}

In this paper, we have proposed to use scalable modulation schemes to provide differentiated services in the PHY layer. We have formulated a cross-layer optimization problem, aimed to maximize the profit of video services by choosing appropriate PHY layer modulation and coding schemes. Extensive simulation results have demonstrated that, with the flexibility of s-mod, we can have better PHY layer configurations to achieve significant profit gains for wireless videocast.

\section{REFERENCES}

[1] T. Cover. Broadcast channels. IEEE Trans. on Information Theory, 18(1):2-14, Jan. 1972.

[2] S. Wang and B. K. Yi. Optimizing enhanced hierarchical modulations. In IEEE GLOBECOM'08, Nov./Dec. 2008.

[3] D. Mitran. Stochastic theory of a fluid model of producers and consumers coupled by a buffer. Advances in Applied Probability, 20(3):646-676, Sept. 1988.

[4] E. Shihab, L. Cai, F. Wan, A. Gulliver, and N. Tin. Wireless mesh networks for in-home IPTV distribution. IEEE Network, 22(1):52-57, Jan.-Feb. 2008.

[5] L. X. Cai, L. Cai, X. Shen, and J. W. Mark. Resource management and QoS provisioning for IPTV over mmWave-based WPANs with directional antenna. ACM/Springer MONET, 14(2):210-219, Apr. 2009.

[6] Q. Du and X. Zhang. Statistical QoS provisionings for wireless unicast/multicast of layered video streams. In IEEE Infocom'09, pages 477-485, Apr. 2009.

[7] R. Cohen, G. Grebla, and L. Katzir. Cross-layer hybrid FEC/ARQ reliable multicast with adaptive modulation and coding in broadband wireless networks. In IEEE Infocom'09, pages 1917-1925, Apr. 2009.

[8] J. Xu, X. Shen, J.W. Mark, and J. Cai. Adaptive transmission of multilayered video over wireless fading channels. IEEE Trans. on Wireless Communications, 6(6):2305-2314, June 2007.

[9] J. She, X. Yu, P. Ho, and E. Yang. A cross-layer design framework for robust IPTV services over IEEE 802.16 networks. IEEE JSAC, 27(2):235-245, Feb. 2009.

[10] L. Cai, Y. Luo, S. Xiang, and J. Pan. Scalable modulation for wireless broadcast. Technical report, Univ. of Victoria, Victoria, BC, Canada, Dec. 2009. Available http://cn.ece.uvic.ca/publication. html\#report. 